\title{
Perfil das internações de crianças por fissuras labiais e/ou palatinas na região
}

\section{Nordeste do Brasil}

\author{
Profile of children's hospitalizations for cleft lip and/or palate in Northeast Brazil \\ Perfil de hospitalizaciones de niños por labio leporino y/o paladar hendido en el noreste de Brasil
}

Recebido: 01/06/2021 | Revisado: 13/06/2021 | Aceito: 04/07/2021 | Publicado: 15/07/2021

\author{
Aliny Thaisy Araújo Costa \\ ORCID: https://orcid.org/0000-0002-9120-7386 \\ Universidade Federal de Campina Grande, Brasil \\ E-mail: thaisyaliny@gmail.com \\ Júlia Kiara da Nóbrega Holanda \\ ORCID: https://orcid.org/0000-0002-1426-0714 \\ Universidade Federal de Campina Grande, Brasil \\ E-mail: juliakiaranh@gmail.com \\ Lara Danúbia Galvão de Souza \\ ORCID: https://orcid.org/0000-0001-5693-9453 \\ Universidade Federal de Campina Grande, Brasil \\ E-mail: lara.danubia@outlook.com \\ Lorena Layanne Pereira Custódio \\ ORCID: https://orcid.org/0000-0002-6379-5993 \\ Universidade Federal de Campina Grande, Brasil \\ E-mail: lorenalayanne888@gmail.com \\ Maria Luiza Dantas da Silva \\ ORCID: https://orcid.org/0000-0001-6694-8388 \\ Universidade Federal de Campina Grande, Brasil \\ E-mail: luizadantasodontologia@gmail.com \\ Debora Lana Alves Monteiro \\ ORCID: https://orcid.org/0000-0002-4731-6737 \\ Universidade Estadual da Paraíba, Brasil \\ E-mail: a.deboralana@gmail.com \\ Ana Karina Almeida Rolim \\ ORCID: https://orcid.org/0000-0001-6910-2898 \\ Universidade Estadual da Paraíba, Brasil \\ E-mail: k_rolim19@hotmail.com \\ Abrahão Alves de Oliveira Filho \\ ORCID: https://orcid.org/0000-0002-7466-9933 \\ Universidade Federal de Campina Grande, Brasil \\ E-mail: abrahao.farm@gmail.com \\ Elizandra Silva da Penha \\ ORCID: https://orcid.org/0000-0001-6264-5232 \\ Universidade Federal de Campina Grande, Brasil \\ E-mail: elizandrapenha@hotmail.com
}

\begin{abstract}
Resumo
As fissuras labiais e/ou palatinas são as malformações congênitas mais comuns da cabeça e pescoço, e ocorrem pela falta de fusão dos processos faciais embrionários, durante o período entre a quarta e a sétima semana de vida intrauterina. Este estudo tem o objetivo de analisar o perfil de crianças internadas com fissuras labiais e/ou palatais na região Nordeste do Brasil, no período de 2015 a 2019. Trata-se de um estudo epidemiológico do tipo observacional e transversal, retrospectivo, descritivo, qualitativo com abordagem documental, no qual foram analisados os casos quanto aos seguintes parâmetros: idade, gênero, cor, caráter do atendimento e distribuição por estado. Os dados foram colhidos no Sistema de Informações Hospitalares do SUS (SIH/SUS) através do DATASUS, lançados em Excel e submetidos a análise estatística no IBM SPSS Statistics for Windows. Foram registradas 6598 internações por fenda labial e/ou palatina. Foi observado que o atendimento de caráter eletivo foi prevalente ao de urgência, sendo a Bahia o estado com maior número de internações quando levado em conta ambos os caráteres. Quanto à idade, gênero e grupo ético-racial, foi constatado que crianças pardas, com idade de 1 a 4 anos e sexo masculino eram as mais afetadas. Foi verificado a ocorrência de 10 óbitos durante o período estudado. Espera-se que este estudo traga informações para o leitor e aponte para a importância da continuidade de pesquisas sobre o tema, oferecendo uma maior difusão sobre a situação médica destes pacientes.
\end{abstract}

Palavras-chave: Anormalidades congênitas; Genética; Odontopediatria; Hospitalização. 


\begin{abstract}
Cleft lip and/or palate are the most common congenital malformations of the head and neck, and occur due to the lack of fusion of embryonic facial processes, during the period between the fourth and seventh weeks of intrauterine life. This study aims to analyze the profile of children hospitalized with cleft lip and/or palate in the Northeast region of Brazil, in the period from 2015 to 2019. This is an observational and transversal, retrospective, descriptive, qualitative epidemiological study with documentary approach, in which the cases were analyzed regarding the following parameters: age, gender, color, character of service and distribution by state. The data were collected in the SUS Hospital Information System (SIH / SUS) through DATASUS, released in Excel and submitted to statistical analysis in IBM SPSS Statistics for Windows. 6598 hospitalizations for cleft lip and / or palate were recorded in the period from 2015 to 2019. It was observed that the attendance of an elective character was prevalent to the emergency, with Bahia being the state with the highest number of hospitalizations when taking into account both characters. As for age, gender and ethical-racial group, it was found that brown children, aged 1 to 4 years and male were the most affected. In addition, 10 deaths occurred during the study period. It is hoped that this study will bring information to the reader and point to the importance of continuing research on the topic, offering a greater diffusion about the medical situation of these patients.
\end{abstract}

Keywords: Congenital abnormalities; Genetics; Pediatric dentistry; Hospitalization.

\title{
Resumen
}

El labio y/o paladar hendido son las malformaciones congénitas más comunes de la cabeza y el cuello, y ocurren por la falta de fusión de los procesos faciales embrionarios, durante el período comprendido entre la cuarta y séptima semanas de vida intrauterina. Este estudio tiene como objetivo analizar el perfil de niños hospitalizados con labio leporino y/o paladar hendido en la región Nordeste de Brasil, de 2015 a 2019. Es un estudio epidemiológico de tipo observacional y transversal, retrospectivo, descriptivo, cualitativo. con enfoque documental, en el cual se analizaron los casos con respecto a los siguientes parámetros: edad, género, color, carácter de servicio y distribución por estado. Los datos se recopilaron en el Sistema de Información Hospitalaria del SUS a través de DATASUS, se publicaron en Excel y se sometieron a análisis estadístico en IBM SPSS Statistics para Windows. Se registraron 6598 hospitalizaciones por labio leporino y/o paladar hendido en el período de 2015 a 2019. La asistencia de carácter electivo fue preponderante a la emergencia, siendo Bahía el estado con mayor número de hospitalizaciones al tomar en cuenta a ambos personajes. En cuanto a edad, género y grupo ético-racial, se encontró que los niños de raza morena, de 1 a 4 años y del sexo masculino fueron los más afectados. Ocurrieron 10 muertes durante el período de estudio. Se espera que este estudio aporte información al lector y señale la importancia de continuar la investigación sobre el tema, ofreciendo una mayor difusión sobre la situación médica de estos pacientes.

Palabras clave: Anomalías congénitas; Genética; Odontología pediatrica; Hospitalización.

\section{Introdução}

As malformações congênitas (MC) são representadas por anomalias funcionais ou estruturais do desenvolvimento fetal, ocasionadas por algum fator anterior ao nascimento, mesmo que este não seja aparente ao nascer e se manifeste tardiamente (Pereira, Bião de Souza \& Santos, 2018). As fissuras labiais e/ou palatinas (FL/P), também conhecidas como fendas orofaciais, são uma das MC mais frequentes e possuem alta incidência, ocorrendo em um a cada 750 recém-nascidos vivos (Lombardo-Aburto, 2017).

As fendas orofaciais podem ser encontradas isoladas ou como parte de uma síndrome. Na literatura, têm-se aproximadamente 400 síndromes que podem ser acompanhadas por malformações como as fissuras. No entanto, o índice de ocorrência de fissuras não-sindrômicas é muito mais alto do que aquelas associadas a esta condição, representando cerca de 70\% a 90\% dos casos (Costa, Takeshita \& Farah, 2013; Sousa \& Roncalli, 2017).

Tais deformidades frequentemente estão relacionadas e estabelecem as malformações congênitas mais comuns da cabeça e do pescoço (Lombardo-Aburto, 2017). São originadas da não junção ou junção incompleta dos processos faciais durante o período entre a quarta e a sétima semana de vida embrionária (Gómez, Villavicencio \& Vilchis, 2015; Lourenço Ribeiro, Teixeira Das Neves, Costa \& Ribeiro Gomide, 2003) e são capazes de atingir diversas estruturas, como: lábio, processo alveolar, palato duro e palato mole. Além disso, podem ser unilaterais ou bilaterais, completas ou incompletas (Lombardo-Aburto, 2017).

Diversos estudos afirmam que a causa dessa anomalia é de natureza multifatorial, tendo os fatores genéticos e ambientais um papel crucial em sua aparência (Ahmed, Bui \& Taioli, 2017; Candotto et al., 2019; Zhang \& Arneja, 2017). Os 
fatores ambientais podem ser físicos, químicos ou biológicos, sendo chamados de teratogênicos por alterarem o desenvolvimento embriológico causando as malformações (Lombardo-Aburto, 2017). Entre esses, temos a deficiência nutricional da mãe, alterações hormonais, substâncias agressivas e tóxicas como álcool, tabaco e medicamentos (ansiolíticos, barbitúricos e fenitoína ou hidantoína, por exemplo), sendo estes últimos os responsáveis por $2 \%$ de todos os defeitos de nascimento (Rubio Álvarez, 2017). Já o fator hereditário está presente entre $20 \%$ a $25 \%$ dos casos como fator dominante, segundo estudos em países da América Central (Mejía \& Suárez, 2012).

As FL/P podem ser classificadas de diferentes formas, com base em aspectos morfológicos e/ou embrionários. A mais adotada no Brasil é a Classificação de Spina, dividida em quatro grupos: fissuras pré-forame incisivo ou, simplesmente, fissuras labiais (FL), fissuras pós-forame incisivo ou fissuras palatinas (FP), fissuras transforame incisivo ou fissuras labiopalatinas (FLP) e fissuras raras da face (Alarcón \& Sá, 2017).

Os indivíduos acometidos por essas fissuras geralmente têm inúmeras alterações que vão desde problemas de alimentação, respiração nasal, audição, crescimento facial, desenvolvimento dental, fonação e estética, comprometendo e dificultando assim funções primordiais. Constituem também um importante problema biológico e psicológico que afeta o núcleo familiar e o ambiente social (Giusti-Barreto, Panchana-Moreira, García-Muñoz \& Zurita-Yong, 2018; Santos, Corrêa, Alves \& Farias, 2019). Assim, para um desenvolvimento neuropsicomotor adequado e melhora da saúde e qualidade de vida de pacientes e suas famílias, a identificação precoce é essencial visando uma intervenção também precoce (Santos, 2019).

O processo de reabilitação de portadores de FL/P tem início com a queiloplastia e palatoplastia, cirurgias plásticas que são realizadas nos primeiros meses e anos de vida, respectivamente. No entanto, o processo reabilitador não se restringe no reparo anatômico da fissura, sendo necessária a atuação de uma equipe multiprofissional e uma abordagem interdisciplinar em épocas oportunas (Freitas e Silva, Mauro, Oliveira, Ardenghi, Bönecker, 2008; Paranaíba, Miranda, Ribeiro, Barros \& Martelli-Júnior, 2011; Razera, Trettene \& Tabaquim, 2016; Silva Filho \& Freitas, 2007).

Como um problema de saúde pública, as FL/P exigem uma terapia de alto custo e complexidade, realizada em centros especializados terciários, representados pelos hospitais de grande porte (Ribeiro \& Moreira, 2012). No Brasil, a distribuição de unidades de saúde habilitadas para realizar esse tipo de procedimento é desigual, com maior concentração na região Sudeste. Consequentemente, muitas pessoas têm que realizar longas viagens para conseguir tratamento médico (Sousa \& Roncalli, 2017).

O perfil de morbidade é um parâmetro importante para a definição das políticas de saúde. A média de permanência hospitalar, considerando-se todas as internações ocorridas de janeiro de 2015 a dezembro de 2019, foi de 2,1 dias no Nordeste. No mesmo intervalo de tempo, as internações hospitalares no Sistema Único de Saúde (SUS), em todo o Nordeste, geraram um custo de quase $\mathrm{R} \$ 11$ milhões (Brasil, 2020a).

Com base no exposto, o objetivo do presente estudo foi analisar o perfil das internações hospitalares de crianças com fissuras labiais e/ou palatinas na região Nordeste do Brasil, notificadas no DATASUS no período compreendido entre os anos de 2015 a 2019.

\section{Metodologia}

O presente trabalho trata-se de um estudo epidemiológico do tipo observacional e transversal, retrospectivo, descritivo, qualitativo com abordagem documental, referente aos casos notificados e diagnosticados de crianças internadas com fissuras labiais e/ou palatais, na região Nordeste do Brasil, no período de janeiro de 2015 a dezembro de 2019, totalizando cinco anos. Foram analisados os seguintes parâmetros: idade, gênero, cor, caráter do atendimento e distribuições por estado, com a finalidade de estabelecer o perfil dos portadores de fendas labiopalatais na região Nordeste do Brasil. Segundo Ludke e 
André (2013), a análise documental pode se constituir numa técnica valiosa de abordagem de dados qualitativos, complementando as informações obtidas por outras técnicas e/ou desvelando aspectos novos de um tema ou problema.

A coleta e a caracterização dos dados foram realizadas por meio do Sistema de Informações Hospitalares do Sistema Único de Saúde (SIH/SUS), no DATASUS (Brasil, 2020a) seguindo os passos: informações de saúde - epidemiológicas e morbidade - morbidade hospitalar do SUS (SIH/SUS) - geral, por local de internação, a partir de 2008 - abrangência geográfica: Brasil por região (Nordeste). Em seguida, foram tabulados no programa Microsoft Excel versão 2016.

O Departamento de Informática do Sistema Único de Saúde (DATASUS) tem por principal objetivo estruturar sistemas de informação em saúde, integrar dados em saúde, bem como auxiliar na gestão dos diversos níveis de atenção em saúde, (Lima, Januário, Lima \& Moura, 2015) sendo um importante meio divulgador de epidemiologia da saúde brasileira.

Após a coleta, os dados foram tratados e analisados estatisticamente por meio do programa IBM SPSS Statistics for Windows, dispondo os resultados na forma de gráficos e tabelas com frequências absolutas e percentuais.

Este estudo foi dispensado de apreciação ética pelo Comitê Permanente de Ética em Pesquisa Envolvendo Seres Humanos sob o ofício $n^{\circ}$ 08/2018, por se tratar de banco de dados de domínio público e irrestrito. Todas as recomendações estabelecidas pelas Resoluções 466/12 - CNS/MS10 e 510/2016 - CNS/MS,11 foram seguidas.

\section{Resultados}

De acordo com dados disponíveis no Sistema de Informações sobre Nascidos Vivos - SINASC (Brasil, 2020b), no período de 2015 a 2018, foram registrados 1.483 nascimentos com anomalia congênita do tipo fenda labial e/ou palatina, de um total de 3.296.654 nascimentos vivos, no Nordeste brasileiro.

Com os resultados obtidos por meio do SIH/SUS, foi possível observar o número de internações por fenda labial e fenda palatina no Nordeste brasileiro nos anos de 2015 a 2019 (Gráfico 1).

Gráfico 1. Distribuição em valores absolutos das internações por fenda labial e/ou palatina em crianças de 0 a 14 anos, no período de janeiro de 2015 a dezembro de 2019.

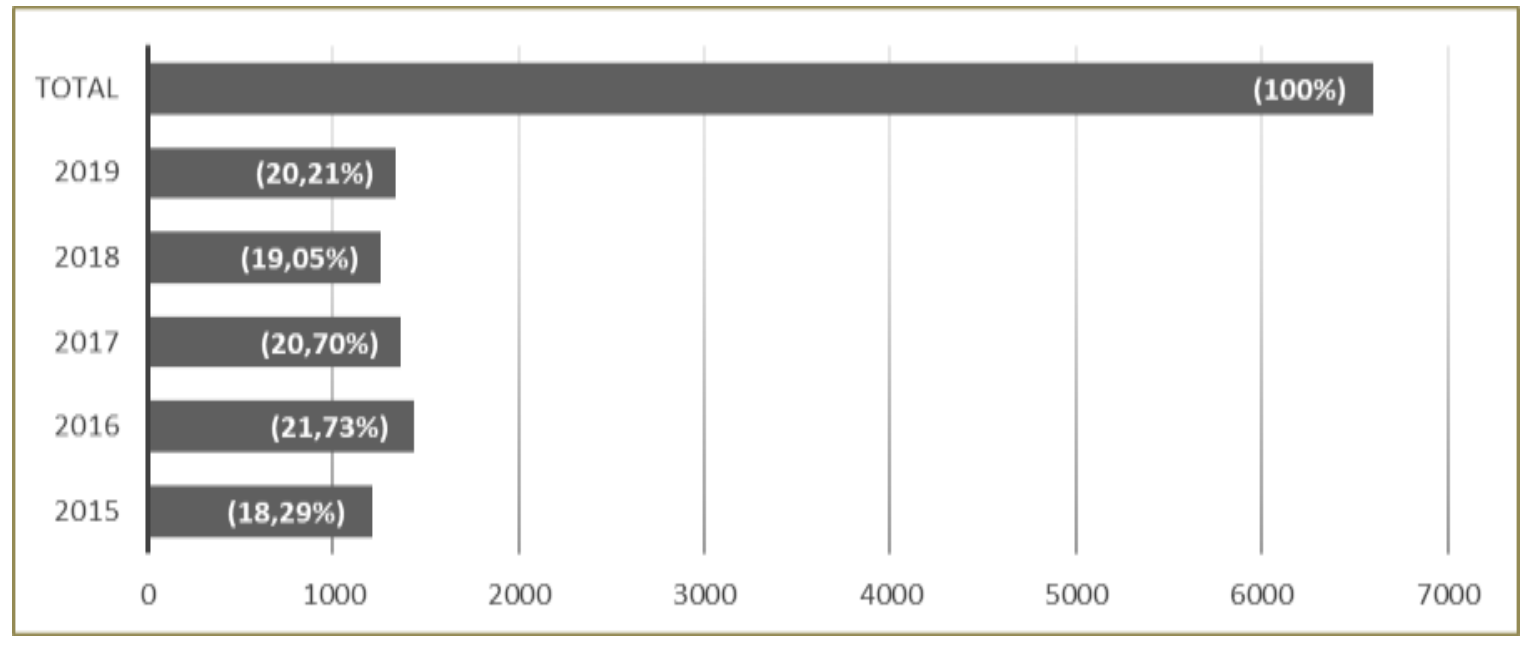

Fonte: Ministério da Saúde - Sistema de Informações Hospitalares do SUS (SIH/SUS).

Quando observado o caráter das internações (Tabela 1), os dados apresentam prevalência daquelas de caráter eletivo, tendo o maior número desses atendimentos ocorrido nos estados de Pernambuco e Bahia. Quanto às internações de urgência, destaca-se o estado do Ceará, apresentando mais da metade dos casos desse caráter $(\mathrm{n}=1096 ; 59,98 \%)$. Dessa forma, considerando ambos os motivos de internações, no intervalo de 2015 a 2019, em primeiro lugar, aparece a Bahia $(\mathrm{n}=1684$; 
25,55\%), seguida de Pernambuco ( $\mathrm{n}=1591 ; 24,12 \%)$, estando o Ceará em terceiro lugar $(\mathrm{n}=1206 ; 18,27 \%)$. O Piauí surge na quarta posição, ainda que com valor expressivamente menor $(n=506 ; 7,66 \%)$. Ademais, verifica-se que os estados de Sergipe e Alagoas respondem pela menor frequência de internações, sejam estas eletivas ou de caráter emergencial.

Tabela 1. Distribuição em valores absolutos e percentuais segundo caráter de internação de crianças de 0 a 14 anos, com fenda labial e/ou palatina, por estado no Nordeste.

\begin{tabular}{cccc}
\hline Unidade da Federação & Eletivo & Urgência & Total \\
\hline Alagoas & $220(80 \%)$ & $55(20 \%)$ & $275(4,16 \%)$ \\
Bahia & $1456(86,46 \%)$ & $228(13,54 \%)$ & $1684(25,55 \%)$ \\
Ceará & $110(9,12 \%)$ & $1096(90,88 \%)$ & $1206(18,27 \%)$ \\
Maranhão & $81(22,56 \%)$ & $278(77,44 \%)$ & $359(5,44 \%)$ \\
Paraíba & $299(88,46 \%)$ & $39(11,54 \%)$ & $338(5,12 \%)$ \\
Pernambuco & $1518(95,41 \%)$ & $73(4,59 \%)$ & $1591(24,12 \%)$ \\
Piauí & $483(95,45 \%)$ & $23(4,55 \%)$ & $506(7,66 \%)$ \\
Rio Grande do Norte & $361(95,25 \%)$ & $18(4,75 \%)$ & $379(5,74 \%)$ \\
Sergipe & $243(93,46 \%)$ & $17(6,54 \%)$ & $260(3,94 \%)$ \\
\hline Total & $\mathbf{4 7 7 1 ( 7 2 , 3 0 \% )}$ & $\mathbf{1 8 2 7}(\mathbf{2 7 , 7 0 \% )}$ & $\mathbf{6 5 9 8}(\mathbf{1 0 0 \%})$ \\
\hline
\end{tabular}

Fonte: Ministério da Saúde - Sistema de Informações Hospitalares do SUS (SIH/SUS).

Quanto ao número de internações por fenda labial e/ou palatina por gênero e faixa etária, verificou-se que, do total de 6598 casos processados nos anos estudados, a maior parte destes $(n=3769 ; 57,12 \%)$, refere-se a fenda labial e/ou palatina em crianças do gênero masculino. Além disso, a faixa etária predominante de ocorrência de internações foi a de 1 a 4 anos $(\mathrm{n}=$ 2921; 44,27\%). Conforme observado, a partir dos 5 anos, o aumento da idade é indiretamente proporcional à frequência de internações, sendo registrada menor ocorrência entre 10 a 14 anos $(n=780 ; 11,82 \%)$ (Tabela 2).

Tabela 2. Distribuição em valores absolutos e percentuais das internações de crianças com fenda labial e/ou palatina no Nordeste, segundo faixa etária e gênero.

\begin{tabular}{lrrr}
\hline Faixa Etária & Masculino & Feminino & Total \\
\hline Menor 1 ano & $1172(59,01 \%)$ & $814(40,99 \%)$ & $1986(30,10 \%)$ \\
$\mathbf{1}$ a 4 anos & $1641(56,18 \%)$ & $1280(43,82 \%)$ & $2921(44,27 \%)$ \\
$\mathbf{5}$ a 9 anos & $517(56,75 \%)$ & $394(43,25 \%)$ & $911(13,81 \%)$ \\
$\mathbf{1 0}$ a 14 anos & $439(56,28 \%)$ & $341(43,72 \%)$ & $780(11,82 \%)$ \\
\hline Total & $\mathbf{3 7 6 9}(\mathbf{5 7 , 1 2 \% )}$ & $\mathbf{2 8 2 9}(\mathbf{4 2 , 8 7 \% )}$ & $\mathbf{6 5 9 8}(\mathbf{1 0 0 \% )}$ \\
\hline
\end{tabular}

Fonte: Ministério da Saúde - Sistema de Informações Hospitalares do SUS (SIH/SUS).

No que se refere ao grupo étnico-racial em que se encaixam essas crianças (Gráfico 2), houve expressivo destaque para crianças pardas $(\mathrm{n}=3465 ; 52,51 \%)$, superando até mesmo o número de internações que não tiveram esse dado informado $(n=2667 ; 40,42 \%)$. Em segundo lugar, encontrou-se a cor branca $(n=251 ; 3,80 \%)$, seguida pela cor preta $(n=162 ; 2,45 \%)$. Por outro lado, crianças amarelas e indígenas foram aquelas com menor predomínio de internações com a anomalia nos dados processados. 
Gráfico 2. Distribuição de crianças de 0 a 14 anos internadas com fenda labial e/ou palatina, segundo cor/raça.

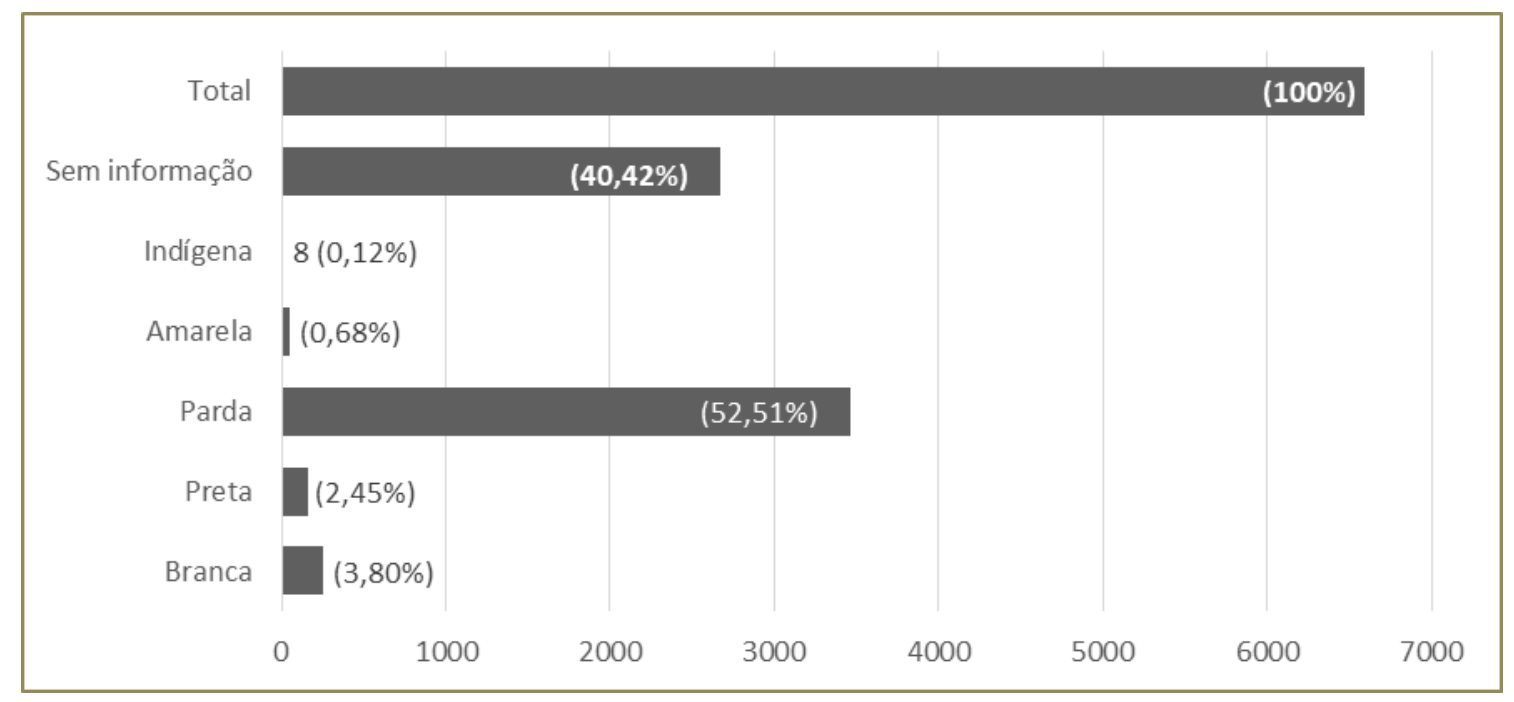

Fonte: Ministério da Saúde - Sistema de Informações Hospitalares do SUS (SIH/SUS).

É necessário destacar, ainda, o processamento de 10 casos de óbito no período de 5 anos estudado, com o ano de 2015 registrando 40\% destes. Verificou-se que 9 casos ocorreram após internações de urgência de crianças menores de 1 ano de idade, enquanto 1 caso se deu após internação de criança entre 1 a 4 anos; 3 dessas ocorrências foram processadas no Piauí, enquanto Pernambuco e Maranhão apresentaram 2 cada, além de 1 caso em Alagoas, Sergipe e Bahia cada.

\section{Discussão}

De acordo com os resultados desta pesquisa, observou-se que o atendimento de caráter eletivo foi prevalente ao de urgência, sendo a Bahia o estado com maior número de internações quando levado em conta ambos os caracteres. No que se referiu ao perfil das crianças internadas com fendas, quanto à idade, sexo e grupo ético-racial, constatou-se que crianças pardas, com idade de 1 a 4 anos e sexo masculino eram as mais afetadas. Além disso, foi verificada a ocorrência de 10 óbitos durante o período estudado.

Conforme o estudo de Shibukawa, Rissi, Higarashi e Oliveira (2019), que analisou tendência e os fatores associados à presença de fenda labial e/ou palatina em recém-nascidos brasileiros e possíveis associações com fatores maternos, assistenciais e da própria criança, no período de 2005 a 2016, a menor taxa média de nascimentos vivos de crianças com fissura labial e/ou fenda palatina foi observada na Região Nordeste com 0,39/1000 nascidos vivos, valor aproximado ao encontrado no SINASC (0,44/1000 nascidos vivos) no período de 2015 a 2018 (Lima et al., 2015).

Segundo Sousa (2017), ao identificar a prevalência de fissuras labiopalatais em brasileiros e os fatores associados ao retardo do tratamento cirúrgico primário da morbidade, no período de 2009 a 2013, as regiões Norte e Nordeste do Brasil apresentaram os piores percentuais de retardo do tratamento, com pretos, amarelos, pardos ou indígenas apresentando uma probabilidade $27 \%$ maior de retardo nas fendas palatinas e $40 \%$ nas fissuras de lábio, evidenciando a desigualdade étnicoracial no acesso ao tratamento. Tais resultados divergem dos dados encontrados no presente estudo, em que a população parda foi a mais prevalente entre as internações no Nordeste, além da cor preta ter apresentado expressiva representatividade, estando em terceiro lugar de ocorrência.

Estudo conduzido por Costa et al. (2013) em uma região do Paraná, apontou a raça branca como a mais expressiva com $81,84 \%$ da amostra de pacientes com fissuras labiopalatais, acompanhada por pardos (12,74\%), negros (3,30\%) e amarelos $(2,12 \%)$. É importante levar em conta que, de acordo com o histórico de padrões de ocupação do território brasileiro, 
resultou-se na maior concentração de pretos e pardos no Norte e Nordeste e brancos no Sul e Sudeste (Instituto Brasileiro de Geografia e Estatística [IBGE], 2011). Portanto, a maior prevalência de FL/P em crianças de cor parda internadas no Nordeste, no presente estudo, pode ter sido influenciada pela forma de distribuição desses grupos étnicos.

Estudos prévios que avaliaram a prevalência de fissuras labiais e/ou palatinas por gênero, observaram uma predominância do gênero masculino, sendo, em média, 60\% mais prevalente em relação ao feminino (Costa et al., 2013; Farinhas, 2017; Santana et al., 2015). Tais achados corroboram este estudo que também apresentou predominância do gênero masculino, evidenciando que, no Brasil, há um acometimento maior de meninos com fissuras labiais e/ou palatinas.

Quando a fenda é estendida ao palato, devido a comunicação buco-nasal, ocorre maior risco de a criança aspirar o alimento, o que pode provocar infecções, pneumonias e otites. Além disso, a fenda pode causar prejuízos no desenvolvimento da fala, linguagem e, havendo dificuldades nos processos alimentares, as anemias também podem se tornar frequentes (Kuhn et al., 2016). Tais acontecimentos podem justificar o número elevado de crianças internadas em caráter emergencial no estudo encontrado. Já no que se refere ao reparo cirúrgico da fenda palatina, podem ocorrer complicações, como sangramento, obstrução respiratória, infecção e deiscência; os dois primeiros ocorrem imediatamente após a cirurgia e, mesmo que raros, requerem reintubação e podem ser fatais (Burg, Chai, Yao, Magee \& Figueiredo, 2016). No presente estudo, entretanto, o motivo de internação e a causa de fatalidade por FL/P não foram analisados devido à ausência dessas informações disponíveis na plataforma DATASUS, sendo importante, então, a sugestão de adição desses dados.

A cronologia cirúrgica preconizada é de realização da queiloplastia primária entre três e seis meses de idade, enquanto a palatoplastia primária deve ocorrer entre 12 e 18 meses (Costa \& Melo, 2019). Tal fato pode justificar a prevalência do processamento de internações de crianças nas faixas etárias menores de 1 ano e entre 1 a 4 anos, de acordo com os dados deste estudo.

Apesar de a utilização de dados secundários nesta pesquisa apresentar as vantagens de fácil acesso com o mínimo de custos, alguns entraves podem dificultar sua coleta, sobretudo no que diz respeito a precisão das informações e possíveis falhas nos registros dos sistemas de informação por parte dos estados. Como retrataram Sousa e Roncalli (2017), o SIH/SUS consegue uma cobertura de aproximadamente $80 \%$ dos dados, havendo exclusão de procedimentos que não são financiados pelo SUS nacional ou aqueles cobertos pelos seguros de saúde, limitando assim o estudo devido ao registro insatisfatório dessas informações. Outro ponto a ser considerado é a falta de distinção entre FL de FP, além da existência de "dados ignorados", subnotificações e a ausência de associação das fendas labiais e/ou palatinas com outros agravos, que diminuem a sensibilidade do estudo e impossibilitam a obtenção de resultados mais precisos (Abreu, Lee, Luquetti \& Starr, 2016).

\section{Conclusão}

De acordo com os resultados encontrados nesta pesquisa, é possível concluir que as internações hospitalares no período de 2015 a 2019 foram constituídas por uma maioria de pacientes do gênero masculino, com faixa etária predominante de 0 a 4 anos. No que se refere à etnia, o estudo demonstrou que crianças de cor parda tiveram mais da metade dos casos de internações, o que se explica pela grande miscigenação da população brasileira.

Foi verificado que o atendimento eletivo se sobressaiu ao de urgência. Porém, constatou-se a ocorrência de 10 casos de óbitos nesse intervalo, principalmente após casos de urgência de crianças menores de 1 ano. Espera-se que este estudo, além de trazer informações para o leitor, aponte a imprescindibilidade da continuidade de pesquisas para o aprofundamento sobre o tema, oferecendo uma maior difusão sobre a situação médica destes pacientes para toda a sociedade.

Assim, como perspectiva de desenvolvimento de trabalhos futuros, sugere-se ampliar o estudo investigando os fatores associados ao desenvolvimento de fissuras orofaciais; avaliar através de dados de caráter quantitativo as crianças acometidas 
que passam por intervenções em tempo hábil; analisar se há o repasse de orientações aos responsáveis pela equipe multiprofissional desde o pré-natal, bem como o impacto deste problema perante a saúde pública.

\section{Referências}

Abreu, M. H. N. G., Lee, K. H., Luquetti, D. V., \& Starr, J. R. (2016). Temporal trend in the reported birth prevalence of cleft lip and/or cleft palate in Brazil, 2000 to 2013. Birth Defects research. Part A: Clinical and Molecular Teratology, 106(9), 789-92.

Ahmed, M. K., Bui, A. H., \& Taioli, E. (2017). Epidemiology of cleft lip and palate. In Designing Strategies for Cleft Lip and Palate Care. IntechOpen.

Alarcón, K. M. G., \& Sá, Á. J. A. (2001). Epidemiological profile of patients with orofacial cleft treated by a reference surgical team in the State of Amazonas, Brazil. Revista Brasileira de Cirurgia Plástica, 32(4), 486-490.

Brasil. (2020a). Departamento de Informática do SUS. Sistema de Informações Hospitalares do SUS - SIH/SUS. Recuperado de http://tabnet.datasus.gov.br.

Brasil. (2020b). Departamento de Informática do SUS. Sistema de Informações sobre Nascidos Vivos - SINASC. http://tabnet.datasus.gov.br.

Burg, M. L., Chai, Y., Yao, C. A., Magee III, W., \& Figueiredo, J. C. (2016). Epidemiology, etiology, and treatment of isolated cleft palate. Frontiers in physiology, 7, 67 .

Candotto, V., Oberti, L., Gabrione, F., Greco, G., Rossi, D., Romano, M., \& Mummolo, S. (2019). Current concepts on cleft lip and palate etiology. Journal of biological regulators and homeostatic agents, 33(3), 145-151.

Costa, B. C., \& Melo, F. D. (2019). Atenção ao bebê com fenda labiopalatina. Trabalho de Conclusão de Curso, Universidade de Taubaté.

Costa, R. R. D., Takeshita, W. M., \& Farah, G. J. (2013). Levantamento epidemiológico de fissuras labiopalatais no município de Maringá e região. Revista da Associacao Paulista de Cirurgioes Dentistas, 67(1), 40-44.

DATASUS (2020). Sistema de Informações Hospitalares do SUS - SIHSUS. http://tabnet.datasus.gov.br.

Farinhas, G. V. (2017). Os sentidos produzidos nos discursos de mães frente ao nascimento de filhos com malformação labiopalatal e os recursos da rede do SUS disponibilizados para o cuidado. Dissertação de Mestrado, Universidade de Santa Cruz do Sul, Santa Cruz do Sul, RS, Brasil.

Freitas e Silva, D. S., Mauro, L. D. L., Oliveira, L. B., Ardenghi, T. M., \& Bönecker, M. (2008). Estudo descritivo de fissuras lábio-palatinas relacionadas a fatores individuais, sistêmicos e sociais. $R G O, 56(4), 387-391$.

Giusti-Barreto, J. C., Panchana-Moreira, G. M., García-Muñoz, T. L., \& Zurita-Yong, G. E. (2018). Caries dental y maloclusiones en infantes con labio y/o paladar hendido. Polo del Conocimiento, 3(9), 15-23.

Gómez, O. V., Villavicencio, M. Á. F., \& Vilchis, M. D. C. V. (2015). Prevalencia de dientes supernumerarios en niños con labio y/o paladar fisurado. Revista odontológica mexicana, 19(2), 81-88.

Instituto Brasileiro de Geografia e Estatística. (2011). Censo demográfico 2010: características da população e dos domicílios: resultados do universo. Rio de Janeiro, RJ: IBGE.

Kuhn, V. D., Miranda, C., Dalpian, D. M., de Moraes, C. M. B., Backes, D. S., Martins, J. S., \& dos Santos, B. Z. (2012). Fissuras labiopalatais: revisão da literatura. Disciplinarum Scientia Saúde, 13(2), 237-245.

Lima, A. C., Januário, M. C., Lima, P. T., \& de Moura, W. (2015). DATASUS: o uso dos Sistemas de Informação na Saúde Pública. Refas-Revista Fatec Zona Sul, 1(3), 16-31.

Lombardo-Aburto, E. (2017). La intervención del pediatra en el niño con labio y paladar hendido. Acta pediátrica de México, 38(4), $267-273$.

Lourenço Ribeiro, L., Teixeira Das Neves, L., Costa, B., \& Ribeiro Gomide, M. (2003). Dental anomalies of the permanent lateral incisors and prevalence of hypodontia outside the cleft area in complete unilateral cleft lip and palate. The Cleft palate-craniofacial journal, 40(2), 172-175.

Ludke, M. \& André, M. E. D. A. (2013). Pesquisas em educação: uma abordagem qualitativa. E.P.U.

Mejía, A. A. C, \& Suárez, V. D. E. (2012). Factores de riesgo materno predominantes asociados con labio leporino y paladar hendido en los recién nacidos. Archivos de investigación materno infantil, 4(2), 55-62.

Paranaíba, L. M. R., Miranda, R. T. D., Ribeiro, L. A., Barros, L. M. D., \& Martelli-Júnior, H. (2011). Frequency of congenital craniofacial malformations in a Brazilian Reference Center. Revista Brasileira de Epidemiologia, 14, 151-160.

Pereira, A., Bião de Souza, M., \& Santos, J. (2018). Tendência temporal das malformações congênitas do sistema nervoso nos últimos quatro anos no Brasil. Revista Pesquisa em Fisioterapia, 8(1), 16-23.

Razera, A. P. R., Trettene, A. S., \& Tabaquim, M. L. M. (2016). O impacto estressor das cirurgias primárias reparadoras em cuidadores de crianças com fissura labiopalatina. Boletim Academia Paulista de Psicologia, 36(90), 105-123.

Ribeiro, E. M., \& Moreira, A. S. C. G. (2005). Atualização sobre o tratamento multidisciplinar das fissuras labiais e palatinas. Revista Brasileira em Promoção da Saúde, 18(1), 31-40. 
Research, Society and Development, v. 10, n. 8, e41510816719, 2021

(CC BY 4.0) | ISSN 2525-3409 | DOI: http://dx.doi.org/10.33448/rsd-v10i8.16719

Rubio Álvarez, L. J. (2017). Defectos del habla relacionados con maloclusiones dentales, en niños con labio y/o paladar hendido no sindrómico en dentición mixta, de la fundación "niños que rien", República Dominicana. Universidad Nacional de Colombia.

Santana, T. M. D., Silva, M. D. D. P., Brandão, S. R., Gomes, A. D. O. C., Pereira, R. M. R., \& Rodrigues, M. (2015). Nascidos vivos com fissura de lábio e/ou palato: as contribuições da fonoaudiologia para o Sinasc. Revista CEFAC, 17(2), 485-491.

Santos, L., Corrêa, G., Alves, K., \& Farias, M. (2019). Perfil de crianças com fissuras labial e palatal: Operação Sorriso. Revista Enfermagem Contemporânea, $8(1), 72-79$.

Santos, N. J. (2019). Tratamento cirúrgico do lábio leporino. Trabalho de Conclusão de Curso (Bacharelado em Odontologia), Universidade de Uberaba, Uberaba, MG, Brasil.

Shibukawa, B. M. C., Rissi, G. P., Higarashi, I. H., \& Oliveira, R. R. D. (2019). Fatores associados à presença de fissura labial e/ou fenda palatina em recémnascidos brasileiros. Revista Brasileira de Saúde Materno Infantil, 19(4), 947-956.

Silva Filho, O. G., \& Freitas, J. A. (2007). Caracterização morfológica e origem embriológica. In Fissuras labiopalatinas: uma abordagem interdisciplinar. São Paulo: Editora Santos.

Sousa, G. F. T. (2017). Fissuras labiopalatinas no Brasil: prevalência e fatores associados ao retardo do tratamento cirúrgico primário no Sistema Único de Saúde. Dissertação de Mestrado, Universidade Federal do Rio Grande do Norte, Natal, RN, Brasil.

Sousa, G. F. T., \& Roncalli, A. G. (2017). Orofacial clefts in Brazil and surgical rehabilitation under the Brazilian National Health System. Brazilian Oral Research, 31, e23.

Zhang, J. X., \& Arneja, J. S. (2017). Evidence-based medicine: the bilateral cleft lip repair. Plastic and reconstructive surgery, 140(1), 152e-165e. 